\title{
A RARE CASE REPORT-RIGHT LUNG AGENESIS WITH DEXTROCARDIA IN A NEONATE
}

Rathnayaka PGRS ${ }^{1}$, Ganewatte $\mathrm{E}^{2}$

${ }^{1}$ Senior Registrar, The Department of Radiology, National Hospital of Sri Lanka, Colombo.

${ }^{2}$ Consultant Radiologist, The Department of Radiology, Lady Ridgeway Hospital, Colombo

Keywords : Lung agenesis, lung aplasia, lung hypoplasia and dextrocardia

Corresponding Author: Rathnayaka PGRS ${ }^{1}$

Copyright: Rathnayaka PGRS ${ }^{1}$

iD https://orcid.org/0000-0002-7230-3102

\section{Background}

There are spectrums of pulmonary underdevelopment of the lung ranging from pulmonary hypoplasia to aplasia and agenesis. This refers to "lung agenesis - hypoplasia complex". Aplasia and agenesis of the lung are rare conditions with average prevalence is about 1 in 100 000. Lung aplasia is usually unilateral and bilateral pulmonary aplasia is invariably lethal ${ }^{1,2}$.

The term "Lung hypoplasia" has been used to describe rudimentary lung and bronchus with airways, alveoli and pulmonary vessels which are deceased in number and size $\mathrm{e}^{1,2}$.

Pulmonary agenesis and aplasia represent two different forms of arrested lung development that result in absence of the distal lung parenchyma. In Pulmonary agenesis there is complete absence of one or both lungs, including bronchi, bronchioles, vasculature, and respiratory parenchyma. In pulmonary aplasia, only rudimentary bronchi are present, which ends as a blind pouch, without pulmonary vessels or respiratory parenchyma ${ }^{1,2,4}$.

Associated congenital abnormalities are frequent, mainly cardiovascular, and has been reported to occur with VACTERL syndrome ${ }^{1,2}$. These associated abnormalities include tracheal stenosis, esophageal atresia, , tracheoesophageal fistula, bronchogenic cysts, patent ductus arteriosus, tetralogy of fallot, and anomalies of the great vessels. ${ }^{1,2,3}$.

\section{Case report}

We present a case of a neonate who developed respiratory distress at birth. Baby delivered by an elective lower segment caesarean section, with a birth weight of $3.2 \mathrm{~kg}$ at a local hospital and indication was previous LSCS. His antenatal history was unremarkable. No swallowing difficulties and meconium passed. Baby was not cyanosed and the absent breath sound noted on the right side. Baby was transferred later to $\mathrm{LRH}$ for further management. 2D ECHO 
revealed dextrocardia with moderate VSD and a small ASD. No PDA. No renal abnormalities seen in the USS.

Chest X ray revealed complete opacification of the right hemithorax with hyperinflation of the left lung with herniation into the right side. Cardiac silhouette was not seen on the left side. Gastric air bubble was seen on the left side and no evidence dilated pharyngeal pouch. No vertebral abnormalities.

CECT Chest revealed complete absence of right lung parenchyma with hyper expanded left lung which herniated into the right side. Right bronchus, right pulmonary artery and the right pulmonary veins were absent. Left pulmonary veins were noted draining to the left atrium. Dextrocardia was present and liver, spleen and the upper abdominal organs were seen in the normal position. No vertebral anomalies.

\section{Discussion}

Unilateral in lung agenesis result from failure of one of the primitive foregut branches to develop during the early, embryonic stage of lung development. Developmental arrest at a later stage results in lobar agenesis.

If balanced growth is not occurred between the two lung buds, then one side will develop normally, and the other will failed to develop partially (hypoplasia) or completely (aplasia) ${ }^{1}$. Clinically these patients are usually symptomatic and present with neonatal respiratory distress as in our case or recurrent chest infections. Rarely it may go unnoticed until later in childhood or adolescence ${ }^{1,2}$. During prenatal ultrasound screening it is usually presented as a hyperechoic hemithorax . However frequently diagnosis is hard to make, but mediastinal shift will usually be a prominent feature ${ }^{2,5}$. CT / MRI of the chest usually confirm the diagnosis and differentiate spectrum of abnormalities ${ }^{1,2}$.

Prognosis depends on the degree of pulmonary involvement, recurrent chest infections, and also the presence of associated anomalies. No associated anomalies found in our case, but chest infections need to be properly managed.

In unilateral aplasia/ hypoplasia , the remaining normal lung hypertrophies and compensate. Mortality rates still exceeds $50 \%$, mainly because of associated malformations. Right side involvement is known to have a bad prognosis due to two main reasons. One is higher association with other anomalies and another is increased risk of disseminating infection with right sided defects. Progressive pulmonary debilitation occurs with recurrent chest infections ${ }^{1,3}$.

\section{Summary}

Lung aplasia and agenesis are rare congenital conditions. It is usually symptomatic and usually present with respiratory distress in neonate. 


\section{References.}

1. Susan E. Wert, "Normal and Abnormal Structural Development of the Lung" Fetal and Neonatal Physiology (Third Edition)Volume 1, 2004, Pages 783-79 Chapter 76

2. Andreas Adam, Adrian k. Dixon, Jonathan H. Gillard, Cornelia M. Schaefer - Prokop, "Grainger and Allison's Diagnostic Radiology". A textbook of medical imaging. $6^{\text {th }}$ edition.

3. Matthew E Abrams MD, Veda L Ackerman MD \& William A Engle MD Perinatal/Neonatal Case Presentation"Primary Unilateral Pulmonary Hypoplasia: Neonate through Early Childhood "- Case Report, Radiographic Diagnosis and Review of the Literature-, pages667670(2004) . Published: 29 September 2004

4. Howard A. Steiner "Aplasia of the Lung" A case report, RSNA, Radiology -:Nov 1 1956https://doi.org/10.1148/67.5.751

5. Jamshid Sadiqi and Hidayatullah Hamidi "CT features of lung agenesis - a case series (6 cases)"BMC Med Imaging. 2018; 18: 37.Published online 2018 Oct 30. doi: 10.1186/s12880018-0281-5

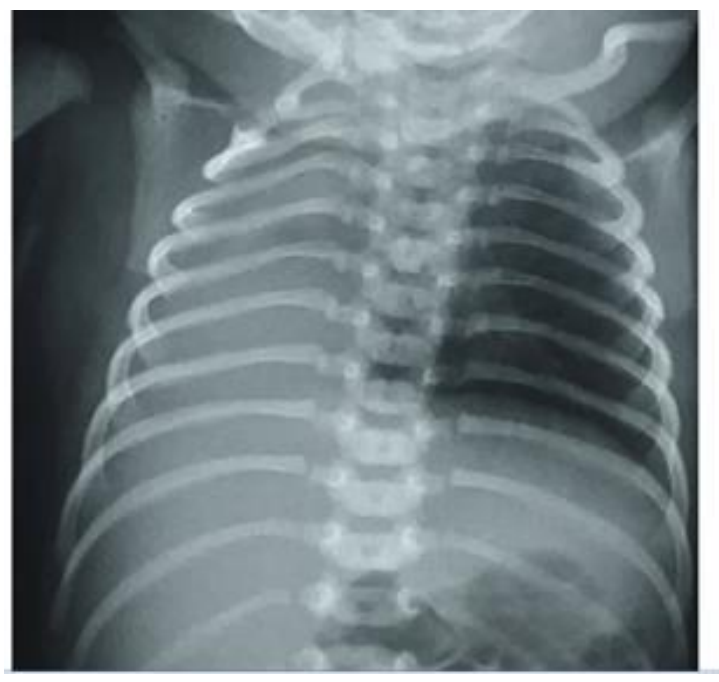

Figure 1- Figure 1-Frontal Chest X-ray of the neonate demonstrate white out right hemithorax. 


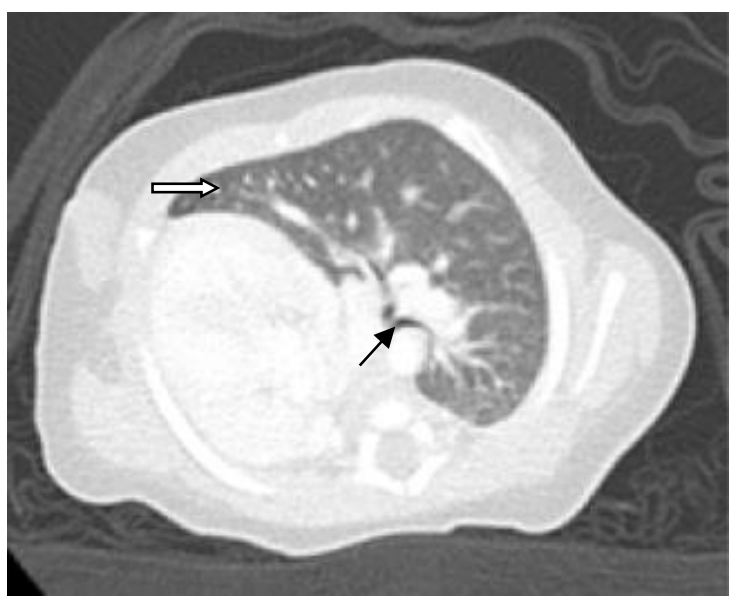

Figure 2- Axial CT images of lung window show absence parenchyma of the right lung with compensatory hyperinflation of the left lung(thick arrow) and absent right bronchus(thin arrow).

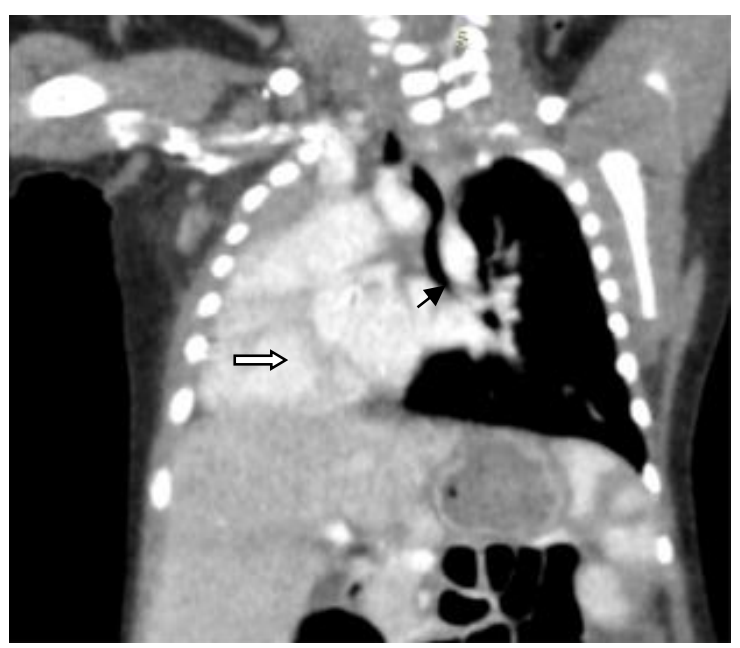

Figure 3- Coronal CECT images show absent right bronchus(thin arrow) and dextrocardia (thick arrow) - Heart occupies the right hemithorax and base apex axis oriented to the right.

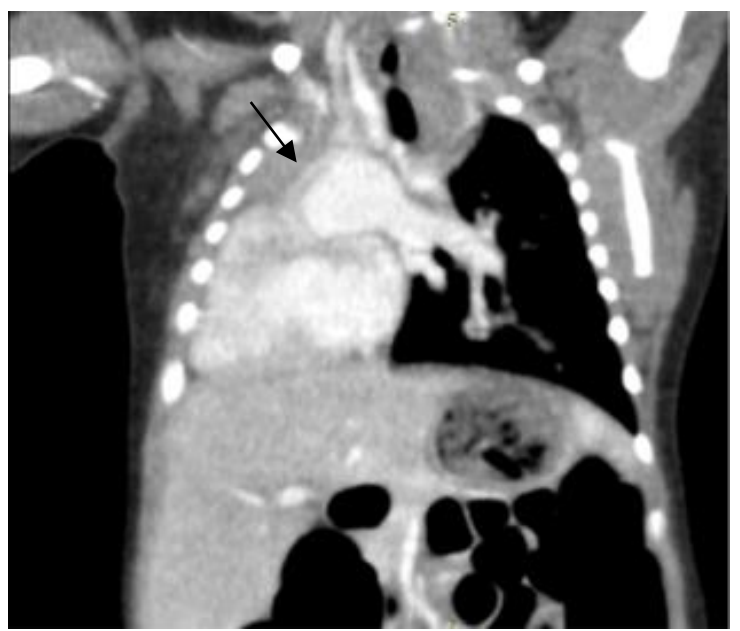

Figure 4- Coronal CECT images show absent right pulmonary artery(arrow). 